\title{
Possible Monitoring and Removal of As(III) by an Integrated System of Electrochemical Sensor and Nanocomposite Materials
}

\author{
Xuan T. Chu, ${ }^{1}$ Quan V. V. Trieu, ${ }^{1,2}$ Thinh Q. Tran, ${ }^{1,2}$ Thanh D. Pham, ${ }^{1}$ Trung Q. Vu, ${ }^{3}$ \\ Thuy H. Tran, ${ }^{1}$ and Tuan A. Mai ${ }^{1,2}$ \\ ${ }^{1}$ Hanoi University of Science and Technology, No. 1, Dai Co Viet Road, Hanoi, Vietnam \\ ${ }^{2}$ National Center for Technological Progress, 25 Le Thanh Tong, Hanoi, Vietnam \\ ${ }^{3}$ Hanoi National University of Education, 136 Xuan Thuy Road, Hanoi, Vietnam \\ Correspondence should be addressed to Tuan A. Mai; mtuan@itims.edu.vn
}

Received 23 May 2018; Accepted 19 August 2018; Published 9 October 2018

Academic Editor: Jana Kukutschova

Copyright (c) 2018 Xuan T. Chu et al. This is an open access article distributed under the Creative Commons Attribution License, which permits unrestricted use, distribution, and reproduction in any medium, provided the original work is properly cited.

In this study, nanocomposites composed of magnetite nanoparticles (MNPs) coated with polyaniline fabricated by in situ polymerization were prepared for arsenic adsorption. Properties of particular MNPs and their nanocomposites were characterized with scanning electron microscopy, X-ray diffraction spectroscopy, and Fourier transform infrared spectroscopy. The As(III) concentration before and after adsorption on nanocomposites was detected by atomic absorption spectroscopy method and then compared with the results measured by a self-developed potentiostat system with anodic stripping voltammetry method. The polyaniline coating resulted in an improvement for As(III) adsorption ability of magnetite nanoparticles, and among the three compositions of PAni/MNP nanocomposites, the $5 \mathrm{wt} \%$ PAni showed the highest capability of As(III) adsorption (or removal) of $50 \mathrm{mg} / \mathrm{g}$. Performing $\mathrm{pH}$ investigation, the concentration of remaining As decreased when $\mathrm{pH}$ increased from 2 to 5 and reached saturation value at higher $\mathrm{pH}$. Above all, the electronic device can be integrated with As(III) removal system using PAni/MNP nanocomposites, proving to act as an independent monitoring system, and even more the adsorbent on the composites could be removed and the recyclability of the material was also investigated.

\section{Introduction}

Arsenite (As III) and arsenate (As V) are arsenic (As) compounds in water which are recognized globally by their extreme health hazards. The acute exposures to arsenic compounds may cause many organ malfunctions (especially the kidney) and several types of cancers [1]. A dose of arsenic which may induce fatal effects for matures is $120-200 \mathrm{mg}$ per $\mathrm{kg}$ body weight (MPBW) and 20 MPBW for infants $[2,3]$. The inorganic As(III) compounds in water are reported to have higher toxicity than the organic ones $[4,5]$. Because arsenic is an ubiquitous element of the outer rock layer, it may naturally contaminate at high levels the groundwater elsewhere [6,7]. In 2010, the World Health Organization (WHO) recommended a maximum contaminant level (MCL) for arsenic in drinking water at $10 \mathrm{ppb}$.
A trace of As(III) in water can be detected by several methods, such as the inductively coupled plasma mass spectroscopy (ICP-MS) or atomic absorption spectroscopy (AAS). However, those instruments are expensive and complicated with long-time sample preparation [8]. Recently, electrochemical method has attracted a large attention of researchers thanks to its sensibility, low cost, and ability to detect very low concentration of analytes $[8,9]$. Electrochemical sensors found its application in DNA identification [10], glucose measurement [11], and environmental monitoring [12]. As for example, As(III) ions were detected by anodic stripping voltammetry (ASV) method with a low limit of detection $[13,14]$. The researchers have kept studying to develop a cost-effective device for on-site heavy metal ion detection in water. Besides, the price of a commercial electrochemical instrument can be up to $\$ 10,000$ or higher and it is 
not suitable for field measurement. In this work, we present a self-developed system on chip- (SOC-) based potentiostat for signal processing of sensors. The circuit was then used to measure As(III) concentration.

Besides, arsenic treatment is highly motivated. Among the materials used for arsenic adsorbents, magnetite nanoparticles (MNPs) provide various possibilities of cost per value reduction as well as removal efficiency. Such material is easily attracted by low magnetization field [15-17] and obtained in a massive amount through some hydrothermal synthesis. In addition, the desorption processes to recycle these MNP adsorbents are possible for several times of use [17]. Therefore, many researchers have focused on MNPs for arsenic adsorbent. However, the problem of using nanosized magnetite for adsorbent is generally laid on their ease of oxidation-induced transformation to maghemite $(\gamma$ $\left.\mathrm{Fe}_{2} \mathrm{O}_{3}\right)$ and hematite $\left(\alpha-\mathrm{Fe}_{2} \mathrm{O}_{3}\right)$. Oxidation together with agglomeration of MNPs often elevates when the size of particles decreases. In addition, the strong magnetic dipole-dipole interaction and Vander Waals attractive forces among the nanoparticles also cause the particles to aggregate [18]. These problems reduce not only the magnetization degree but also the affinity of magnetite toward arsenic $[19,20]$. To maintain adsorptive functionality of MNPs under a corrosive condition, numerous manipulation methods have been proposed, including surfactant-supported nanosuspension and polymer coating $[21,22]$. Among those, nanocomposite of MNPs with polyaniline (emeraldine base coating) has been extensively studied in syntheses and applications for medical and environment purposes. Compared to many coating polymers, polyaniline (PAni) is highly compatible in bio- and environment applications due to its good environment stability, low monomer cost, ease of syntheses and purification, and convenient degrading process $[23,24]$. The PAni/MNP nanocomposite can also be mixed with other polymer substrates such as poly(p-hydroxyaniline) and polyacrylonitrile to increase hydrophilicity of materials in aqueous media and mechanical strength of materials in product processing $[24,25]$. Nevertheless, it is noticeably remarked that there has been no study informing PAni-coated MNPs to be an As(III) adsorbent as well as performing recyclability of this material in metal ion adsorption.

In this study, we focused on synthesis of PAni-coated MNPs for As(III) adsorption. The morphology and recyclability of nanocomposites were evaluated. The detection of As(III) was conducted with the ASV method using a selfdeveloped potentiostat and paired with AAS methods.

\section{Materials and Methods}

2.1. Chemicals. Iron(II) chloride tetrahydrate $\left(\mathrm{FeCl}_{2} \cdot 4 \mathrm{H}_{2} \mathrm{O}\right)$, iron(III) chloride hexahydrate $\left(\mathrm{FeCl}_{3} \cdot 6 \mathrm{H}_{2} \mathrm{O}\right)$, sodium arsenite $\left(\mathrm{NaAsO}_{2}\right)$ as an $\mathrm{As}$ (III) compound, aniline, isopropanol, $\mathrm{HCl}$ solution, phosphate-buffered saline ( $\mathrm{PBS}, \mathrm{pH}=7.4)$, and ammonium persulfate were purchased from Sigma-Aldrich (Missouri, USA). Ammonium hydroxide solution (25\% $\mathrm{NH}_{3}$ in $\mathrm{H}_{2} \mathrm{O}$ ) was purchased from Kanto (Shanghai, China). Deionized (DI) water was produced by deionizer system EW01503-20, Cole Parmer (Illinois, USA), with a resistivity of
$17 \mathrm{M} \Omega \cdot \mathrm{cm}$. Solutions of $1 \mathrm{ppm}$ As(III) were prepared as stock solutions. DI water, prepared solutions, and solid materials were kept in nitrogen gas atmosphere before use. $\mathrm{HCl}$ and PBS solutions were prepared to control the $\mathrm{pH}$ of tested solutions in the range of $2-8$ at room temperature.

2.2. Preparation of Polyaniline/MNP Nanocomposites and Magnetite Nanoparticles (MNPs). $5 \mathrm{ml}$ of $0.4 \mathrm{M} \mathrm{FeCl}_{3}$ and $10 \mathrm{ml}$ of $0.3 \mathrm{M} \mathrm{FeCl}_{2}$ aqueous solutions were mixed in the rubber-plugged flask with nitrogen gas flow. The mixing solution was agitated for 10 minutes at $70^{\circ} \mathrm{C}$. After that, an excessive amount of ammonia for the final $\mathrm{pH}$ of 13 was quickly dropped into the flask to get black precipitated MNP product. Reaction solution was centrifuged to obtain the deposition; the supernatant was discarded. The final deposition part was rinsed 6 times by DI water, being centrifuged and kept in deoxygenated DI water.

A predetermined amount of MNPs was mixed with DI water (100 weight equivalent amount) and isopropanol (20 weight equivalent amount) and then kept in a sonicator at room temperature for 1 hour to homogeneously disperse the MNPs. A predetermined amount of aniline hydrochloride was added into the prepared suspension and magnetically stirred for 1 hour. Three different weight equivalences of aniline monomer (5\%,10\%, and 15\% versus MNPs) were used for coating. The oxidant $\left(\mathrm{NH}_{4}\right)_{2} \mathrm{~S}_{2} \mathrm{O}_{8}$ and $\mathrm{HCl}$ were added into the reaction flask with a molar ratio equivalent to 1.25 and 0.25 of aniline monomer. All solutions were cooled down to $4^{\circ} \mathrm{C}$ in an ice bath and kept under deoxygenated atmosphere. The reaction was carried out for 20 hours in nitrogen gas flow. After the reaction, the mixture was centrifuged and rinsed 5 times by DI water then rinsed in acetone for 2 times to remove water. Finally, the obtained greenish black powders were dried in a vacuum at $40^{\circ} \mathrm{C}$ for 2 hours.

\subsection{Characterization of MNPs and Polyaniline/MNP} Nanocomposites. The shape and size distribution of MNPs as well as the nanocomposites were observed by scanning electron microscopy (SEM) (GeminiSEM, ZEISS Germany). The magnetic property was measured by a vibrating-sample magnetometer DMS 880 (ADE Technologies, USA). The standard crystal structure of $\mathrm{Fe}_{3} \mathrm{O}_{4}$ was confirmed by X-ray diffraction spectroscopy (XRD) (D2 PHASER 2nd, Bruker Singapore Pte. Ltd.). The formation of PAni and nanocomposites was investigated by Fourier transform infrared spectroscopy (FTIR) (Spectrum One, PerkinElmer, Massachusetts, USA). The ions compositions of resulted solutions were confirmed by AAS (iCE 3400 AA, Thermo Fisher Scientific, Massachusetts, USA).

2.4. Arsenic Adsorption Ability and Recyclability Evaluation. Adsorption experiments were performed under deoxygenated atmosphere. $1 \mathrm{mg}$ of each adsorbent was stuffed into a glass tube container $(2 \mathrm{~cm}$ in length, $0.5 \mathrm{~cm}$ in diameter). $1 \mathrm{ml}$ arsenite solutions (As III) of different concentrations were injected into the containers for immersion. The $\mathrm{pH}$ range in this study for arsenic adsorption test was investigated from 2 to 8 , and the adsorption time was from 10 to 60 minutes. Adsorbent containers were sealed by rubber 


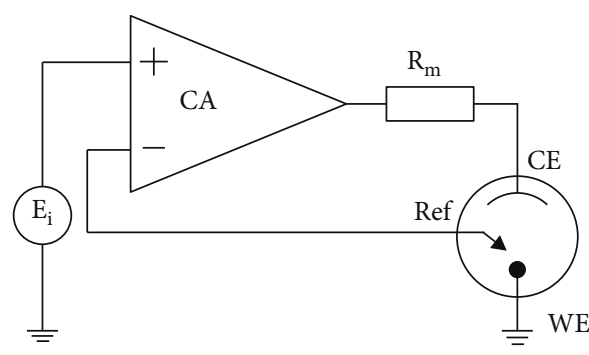

(a)

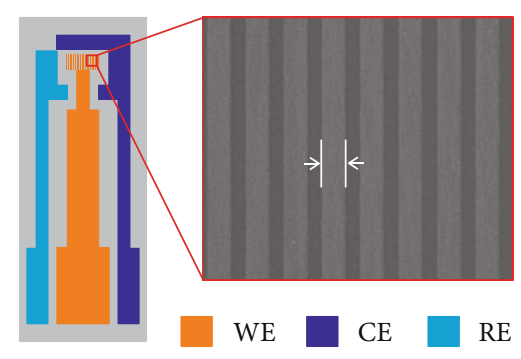

(b)

FIgURE 1: (a) Concept of 3-electrode measurement sensor; (b) electrode configuration and the inset showing the SEM image of WE.

plugs and shaken horizontally by an autoshaker. After immersion time, a cotton fiber was used to replace rubber plugs and the resulted As solutions were moved out by nitrogen gas flow through adsorbent containers. The resulted solution samples were filtered through a $0.22 \mu \mathrm{m}$ syringe filter with mixed cellulose ester membrane (Millipore, USA), and then the final As(III) concentrations in the filtrates were determined by AAS analyses. To perform a desorption experiment, the adsorbent container was washed by $1 \mathrm{ml}$ acetone and immersed in free-arsenite aliquot $(\mathrm{pH}=3)$. The containers were sealed by rubber plugs and shaken for 12 hours. Released As solutions were filtered with the abovementioned procedure and taken into AAS analyses. To evaluate the maximum adsorption ability of materials, each $1 \mathrm{~g}$ adsorbent was immersed in a 11 aqueous solution of $1 \mathrm{ppm}$ As(III) for 20 minutes.

2.5. Detection of Arsenic Ions Using ASV and Electrochemical Sensor. The electrochemical sensor was designed in tripleelectrode configuration: working electrode (WE), reference electrode (RE), and counter electrode (CE) (Figure 1). During the data acquisition, the voltage between the WE and $\mathrm{RE}$ was kept stable and the current generated at the WE was measured. The self-fabricated sensor is illustrated in Figure 1(b), in which all three electrodes were made of gold and the working area of the WE is parallel assembled wires of $2 \mu \mathrm{m} \times 400 \mu \mathrm{m} \times 0.1 \mu \mathrm{m}$ each.

The ASV has a great advantage in heavy metal detection because of its specificity and high sensibility. Apart from arsenic, this method can also be used to detect copper ion $\left(\mathrm{Cu}^{2+}\right)$ or antimony ion $\left(\mathrm{Sb}^{3+}\right)$ [26]. Anodic stripping voltammetry (ASV) measurement includes accumulation and stripping phases. In a sample solution containing As(III), at the accumulation step, the As(III) deposited on the WE under the attractive force due to applied negative voltage and reduced to $\mathrm{As}(0)$. In a latter stripping phase, all accumulated metals will be stripped off the surface of the WE and oxidized, resulting in an electrochemical current peak which is proportional to the amount of adsorbed metal ions on the WE under the accumulation phase as well as the concentration of ions in the solution.

Firmware for programed measurement was written in C for Cypress CY8C27443. The integrated chip can provide a digital-to-analog converter (DAC) up to 9 bits and analogto-digital converter (ADC) up to 14 bits. Text liquid crystal digit (LCD) screen and buttons were used for user interface.
The DAC signal was provided for the electrochemical reaction, then the generated signal was processed and came back to the microcontroller via ADC. The raw signal was then converted into digital and transmitted to a computer via the universal asynchronous receiver/transmitter (UART) protocol.

The hardware is the heart of the design. The circuit diagram of the system was shown in Figure 2. For simplicity, it was built from instrumentation amplifiers (INA) combined with operation amplifiers (OPA). The power is supplied from a linear regulator or USB port. The circuit uses an instrumentation amplifier to measure the current produced at the WE via a sensing resistor. The value of the sensing resistor as well as the gain of instrumentation amplifier can be adjusted so that the measured current range can be changed. Highquality instrumentation amplifier is advantageous over OPA transimpedance amplifier due to low DC offset, low drift, low noise, and high common mode rejection ratio which are suitable for high-precision applications. By using INA and careful processing, the resultant current in nA range can be measured. The voltage applied in the accumulation stage at the WE is negative referred to RE, so that the DAC signal must go through a negative shift and then the processed signal must be leveled up to match with the allowed input range of ADC. The offset voltage was also provided by a channel of microcontroller. The connection of operational amplifier $(\mathrm{OA})$ to the $\mathrm{RE}$ was to ensure that there is no current that comes in or out of the electrode which will make the electrochemical system unstable $[27,28]$. The excitation signal from the OA will be injected to the CE; the inverted form of applied voltage will appear at the WE due to the negative feedback loop between electrodes [29]. To avoid the noises from the environment and inside of the system's setup, some low-pass filter (LPF) had been used to decrease the influence of unwanted signal.

Raw ADC signal was transmitted directly to the computer via the UART protocol. The program was written in LabVIEW (National Instruments Corporation, Austin, Texas, USA) which shows the responding current vs. time in seconds.

\section{Results and Discussion}

3.1. Characterization of Adsorbents. SEM images of bare MNPs and of PAni/MNPs with 5\%, 10\%, and 15\% aniline monomer are shown in Figure 3. Magnetite was formed in a coprecipitation with increasing temperature under 


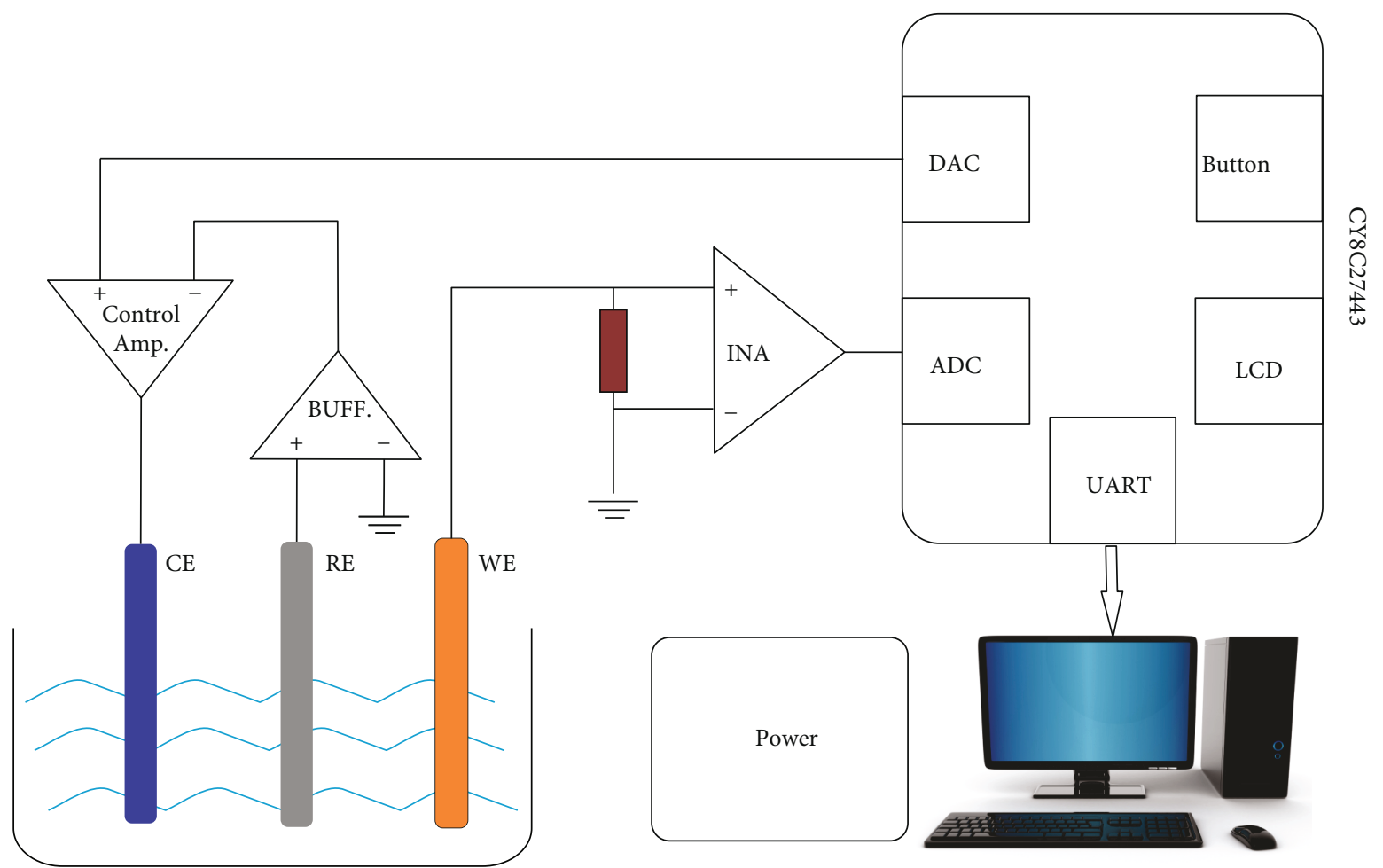

FiguRE 2: Block diagram of potentiostat.

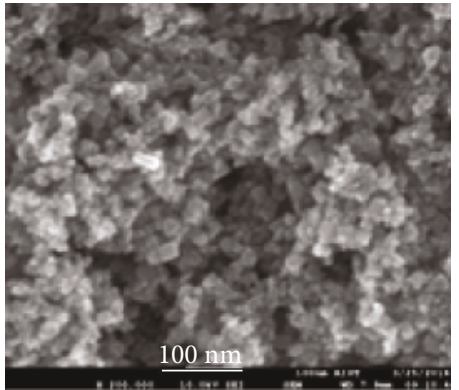

(a)

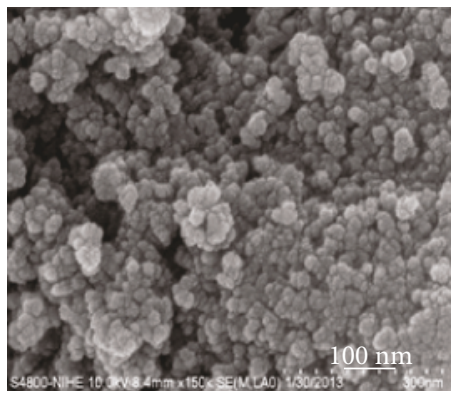

(c)

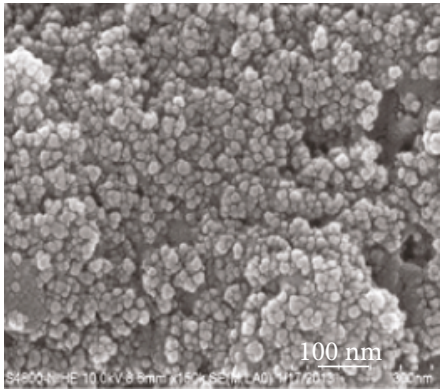

(b)

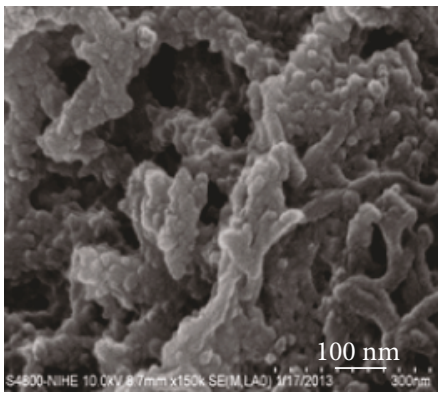

(d)

Figure 3: SEM images of (a) bare MNPs, (b) 5\% PAni/MNPs, (c) 10\% PAni/MNPs, and (d) 15\% PAni/MNP nanocomposites.

aggressive agitation, then followed by sonication resulting in nanoparticles with diameter from 8 to $12 \mathrm{~nm}$ (Figure 3(a)). MNPs were routinely stored in deoxygenated DI water before each characterization and syntheses, to maintain the dispersion and minimize the prospect of being oxidized. The morphologies of nanocomposites were differentiated by the feeding ratios of aniline salt monomer versus MNPs. The particles of nanocomposite with $5 \%$ of aniline monomer were formed in round shape with a diameter range of 15$30 \mathrm{~nm}$ (Figure 3(b)); meanwhile, with 10\% aniline monomer, 


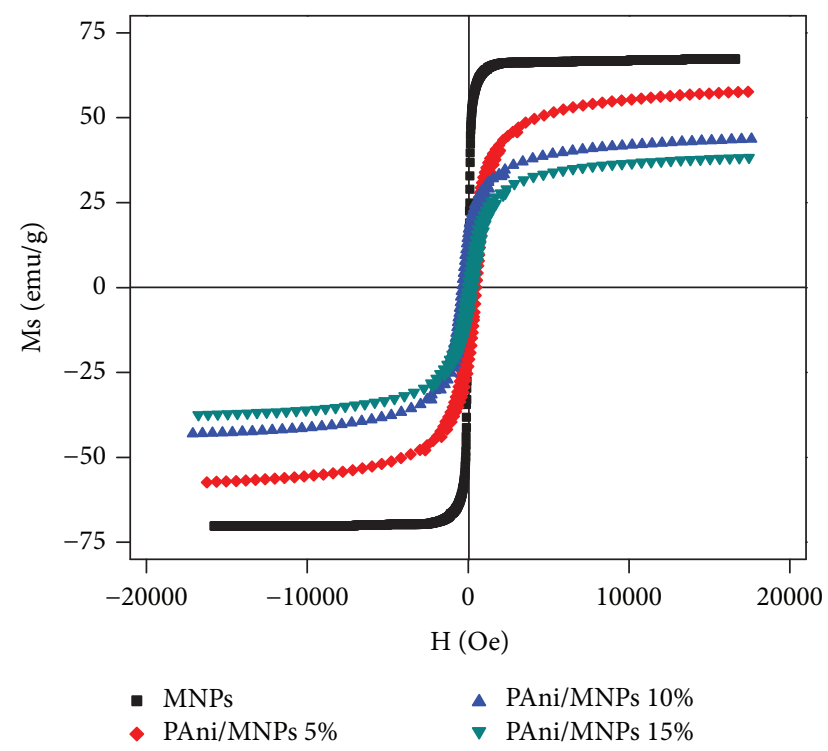

FIgURE 4: Magnetization momenta of MNPs and PANi/MNPs with deferent weight equivalences of aniline monomer.

the nanoparticles were bonded to form a building-flock shape (Figure 3(c)). In a nanocomposite with $15 \%$ aniline monomer feed, it is difficult to see the separation of particles because the nanoparticles, nanoflocks, and short nanofibers are located in a thick polymer matrix (Figure 3(d)).

Together with morphology, the magnetic property of PAni/MNPs was also investigated in order to reveal the relation between nanocomposites' structure and their adsorption ability. As shown in Figure 4, the magnetization momenta of nanocomposites decreased as the composition of coating PAni increased. The reduction of magnetization momenta corresponds to larger radii of nanounits in nanocomposite materials, which was caused by the coverage of PAni layer, while the size of magnetization centers (MNPs) was not changed. Due to higher magnetic field dwelling, the SEM image contrast of bare MNPs (Figure 3(a)) was lower than that of PAni/MNPs (Figures 3(b)-3(d)). By coating with PAni, the agglomerations of particles were supposed to declined, leading to higher surface area ratio of nanocomposites. The magnetization reduction at a particle surface as well as charge elevation at the surface of nanocomposites may lead to an antiagglomeration effect of the PAni layer [30, 31].

Figure 5 shows the XRD patterns of MNPs and PAni/ MNPs showing 7 peaks which characterized for magnetite crystal of $111,220,311,400,422,511$, and 440 planes. No peak of hematite was observed in the XRD spectrum [32] meaning that the obtained nanoparticles contain maghemite or magnetite.

Direct oxidative polymerization of PAni had been performed with three aniline monomer compositions. The FTIR spectra (Figure 6) of PAni, bare MNPs, and PAni/MNP nanocomposites were shown in Figure 6. In the case of PAni, the peaks at $1600,1480,1300$, and $1172 \mathrm{~cm}^{-1}$ are attributed to $\mathrm{C}=\mathrm{C}$ stretching of the quinoid ring, $\mathrm{C}=\mathrm{C}$ stretching of the benzenoid ring, $\mathrm{C}-\mathrm{N}$ stretching of the benzenoid unit, and $\mathrm{N}=$ quinoid= $\mathrm{N}$ vibration [33], respectively. The spectrum of

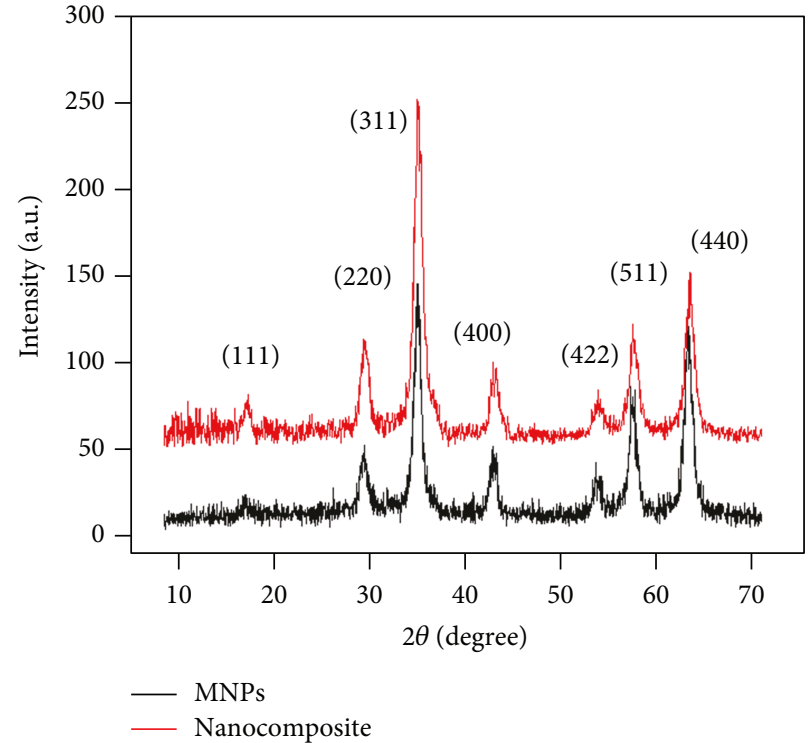

FIgURE 5: XRD patterns of bare MNPs and 5\% PAni/MNP nanocomposite.

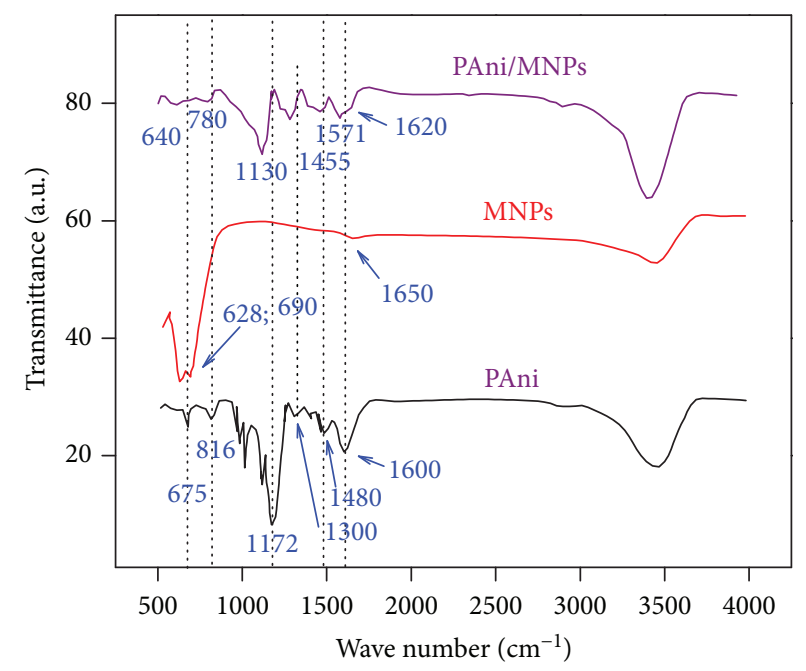

FIgURe 6: FTIR spectra of PAni, bare MNPs, and 5\% PAni/MNP nanocomposites.

bare MNPs exhibits characteristic peaks at 3450 and $1650 \mathrm{~cm}^{-1}$, which can be assigned, respectively, to $\mathrm{O}-\mathrm{H}$ stretching and $\mathrm{O}-\mathrm{H}$ bending vibration of water molecule existing in the physical adsorption or ferrofluid [30]. The strong peaks at 690 and $628 \mathrm{~cm}^{-1}$ are attributed to the stretching vibration mode of $\mathrm{Fe}-\mathrm{O}$ bonds in the crystalline lattice of $\mathrm{Fe}_{3} \mathrm{O}_{4}$. The spectrum of PAni/MNP composite exhibits almost the same vibrational bands as pure PAni, but characteristic peaks shifted to lower wave number, e.g., from 1600 to $1571 \mathrm{~cm}^{-1}$ with $\mathrm{C}=\mathrm{C}$ stretching of the quinoid ring. The shifting of the peaks can be attributed to the interaction of $3 \mathrm{~d}$ orbit of ferrite with nitrogen atoms in PAni to form coordinate bonds [30], which is similar to the band shift on the FTIR spectrum of metal-ligand complexes [34]. Therefore, the shoulder peak around $1620 \mathrm{~cm}^{-1}$ on the 


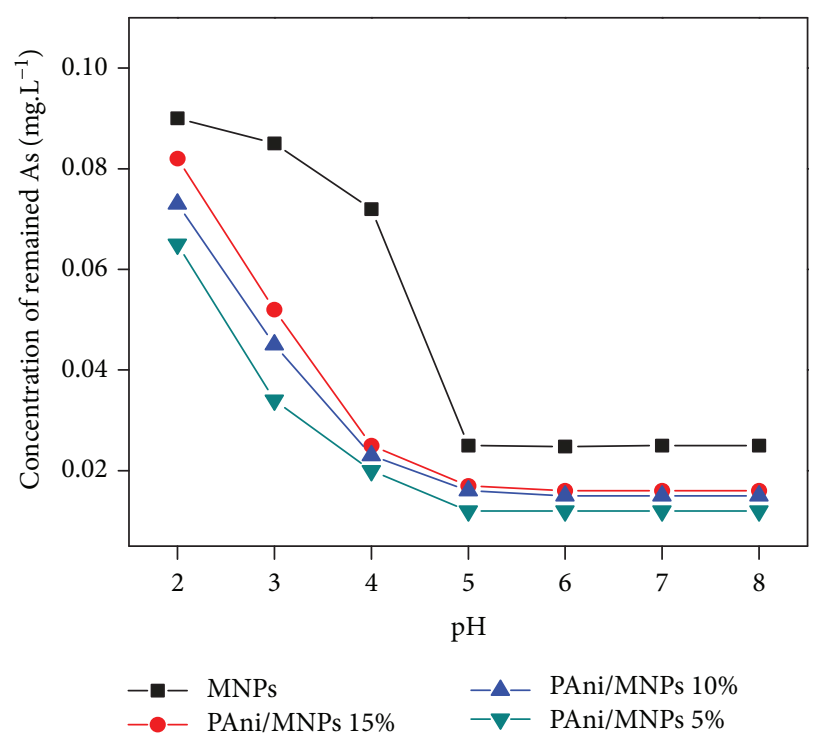

(a)

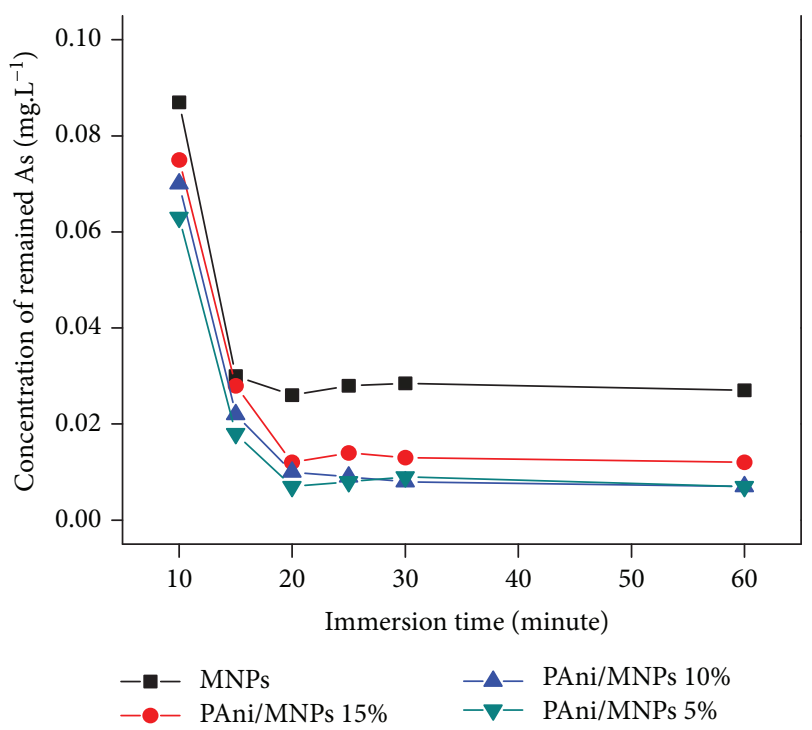

(b)

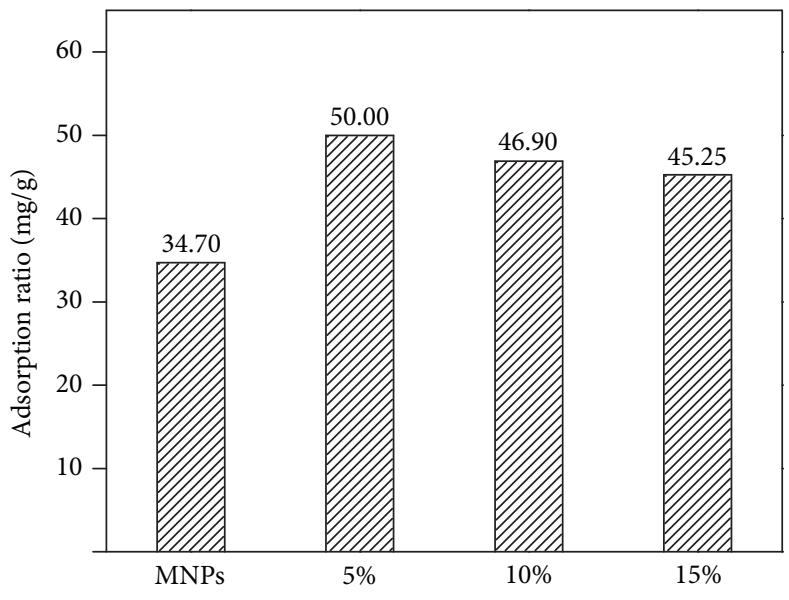

(c)

FIgure 7: Adsorption behavior of PAni/MNPs over (a) pH in 20 mins and (b) immersion time at pH 5; (c) histogram presents maximum $\mathrm{As}(\mathrm{III})$ adsorption ratios of MNPs and PAni/MNP nanocomposite at an optimized condition.

spectrum of the composite is attributed to $\mathrm{O}-\mathrm{H}$ vibration of water molecules that exists in hydrogen bonding with $\mathrm{N}-\mathrm{H}$ groups of PAni. The characterization peak can be explained by two reasons. Firstly, the hydrogen bonding interaction leads to the slight shift of $\mathrm{O}-\mathrm{H}$ vibration to a lower wave number. Secondly, hydrogen-bonded $\mathrm{OH}$ groups also have broader adsorption bands [34]. These results also demonstrate that the coating of PAni to the surface of the magnetic particles occurs through the hydrogen bonding between $\mathrm{N}-\mathrm{H}$ groups in the PAni and $\mathrm{OH}$ group of bonded water molecules in the ferrofluid, leading to the encapsulation of magnetic particles by PAni [30].

3.2. Adsorption Ability of PAni/MNP Nanocomposites. Maximum adsorption values of the adsorbent were examined by immersing each adsorbent in excessive 1 ppm As(III) solution and followed by the previously described immersing steps. As shown in Figure 7(a), the concentration of the remaining As(III) decreased when the $\mathrm{pH}$ increased from 2 to 5 and reached a saturation value at higher $\mathrm{pH}$. In investigating the $\mathrm{pH}$ range, the 5\% PAni composite showed the highest adsorption ability toward As compared to $10 \%$ and 15\% PAni ones and MNPs, too. The similar result was found when varying adsorption time (Figure 7(b)), and the As(III) adsorption reached saturation state after 20 minutes. A histogram of As(III) adsorption ratios of MNPs and PAni/MNPs nanocomposite at optimized condition was illustrated in Figure $7(\mathrm{c})$, in which the maximum adsorption ratio was attained with the sample of 5\% PAni/MNP composite. The adsorption was improved because of the tolerating effect of electron-rich phenyl rings on the side chain of PAni, which enhanced the affinity of Fe(II) in MNPs [30]. It is supposed that PAni may interact with MNPs in a dynamic model, by which the polymer layer partly covers the MNP surface, allowing a slight planar movement of some fragments on each other. Diffusion of As(III) to the MNP surface could be available with suitable distances between MNP and PAni. According to the reports of Liu et al. [35] and Yang et al. [36], 


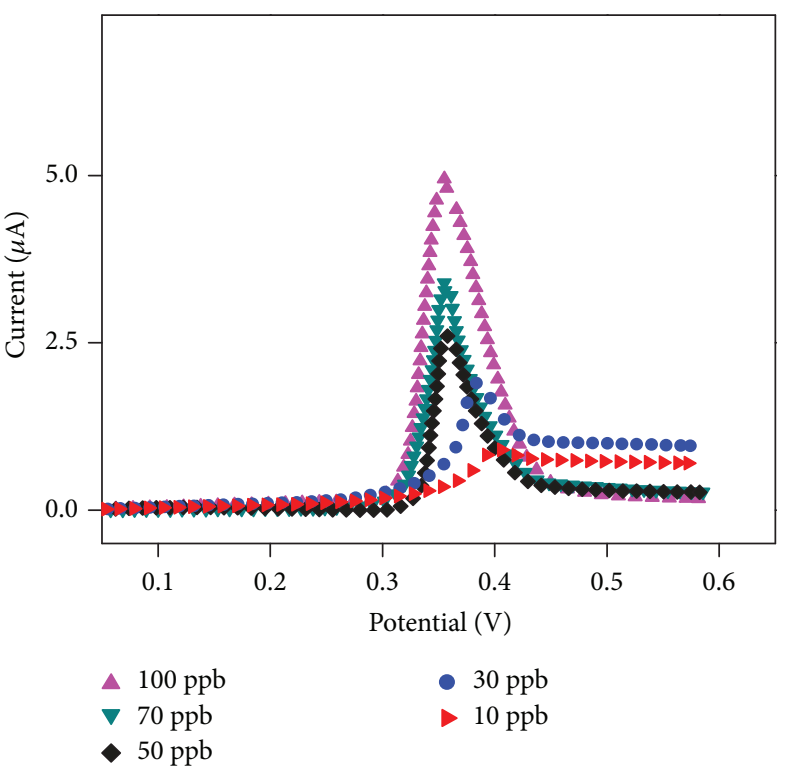

(a)

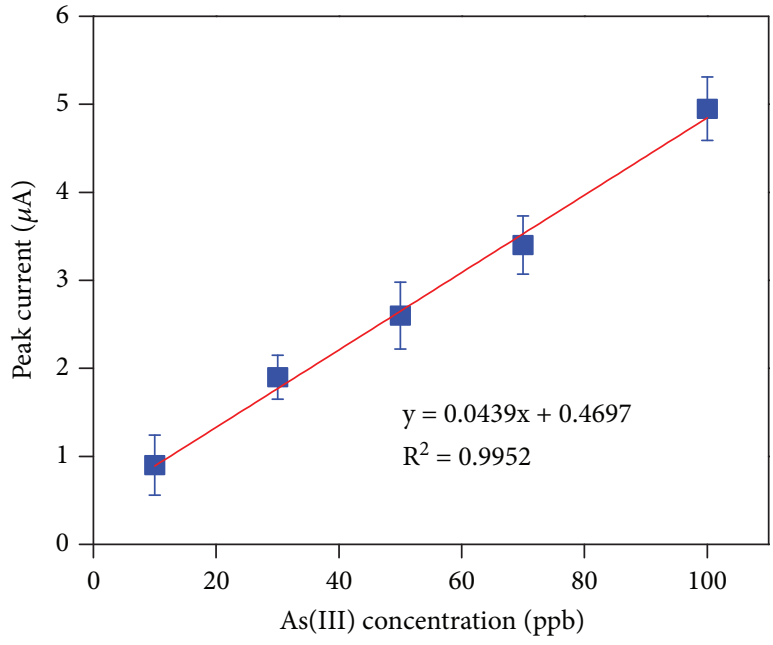

(b)

FIGURE 8: (a) ASV curves of different As(III) concentrations; (b) dependence of peak current versus As(III) concentration in ASV measurement.

the oxidation of MNPs might also be suppressed by the interaction of electron-rich PAni layer on the MNP surface. Besides, in an acidic medium, the amine (-NH-) units of PAni might act as competitive factors to attract the protonated species from MNPs' surfaces [30].

3.3. Operation of Developed Potentiostat Circuit in As(III) Measurement. A portable potentiostat circuit along with the electrode system was used to determine As(III) concentration in the solution as follows: (1) stripping stage at $-0.45 \mathrm{~V}$ for $100 \mathrm{~s}$; (2) stability stage at $-0.3 \mathrm{~V}$ for $10 \mathrm{~s}$, and (3) applied potential then jump to stripping voltage at $0.6 \mathrm{~V}$ at the rate of $1 \mathrm{~V} / \mathrm{s}$. The mentioned potential is between the $\mathrm{WE}$ and $\mathrm{RE}$. The results measured with our potentiostat are shown in Figure 8. The portable potentiostat can measure the trace of As(III) at the concentration of $10 \mathrm{ppb}$, which is the critical level suggested by the WHO. The corresponding peak current and measured concentration of As(III) are a first order and linear function (Figure 8(b)). For each As(III) concentration, three ASV measurements were performed similarly using three separate sensors. Using the developed calibration curve, As(III) concentrations of posttreatment as well as desorption were evaluated during real-time operations of adsorbents.

3.4. Evaluation for Adsorbent's Adsorption, Desorption, and Corrosion. Resulted As solutions after adsorption at $\mathrm{pH}=2$ and $\mathrm{pH}=5$ were used as samples for simple measurement by developed ASV sensing system; the second batch of each solution was separated for confirmation by AAS measurement. Quantitative results of measurements were compared in Table 1. At a higher As(III) concentration from 70 to $100 \mathrm{ppb}$, the ASV sensing system showed high accuracy with
TABle 1: Adsorption behavior of MNPs and PAni/MNPs after 20 minutes. The analysis results obtained by developed ASV sensing system and AAS.

\begin{tabular}{lccccc}
\hline Adsorbent & \multicolumn{2}{c}{$\begin{array}{c}\text { Remained As } \\
\text { conc. by ASV } \\
(\mathrm{mg} / \mathrm{l})^{\mathrm{a}}\end{array}$} & \multicolumn{2}{c}{$\begin{array}{c}\text { Remained As } \\
\text { conc. by AAS } \\
(\mathrm{mg} / \mathrm{l})^{\mathrm{b}}\end{array}$} & Difference \\
& $\mathrm{pH}=2$ & $\mathrm{pH}=5$ & $\mathrm{pH}=2$ & $\mathrm{pH}=5$ & \\
\hline MNPs & 0.094 & 0.025 & 0.091 & 0.028 & $3.2-10.7 \%$ \\
$5 \%$ PAni & 0.085 & 0.010 & 0.082 & 0.009 & $3.6-10.0 \%$ \\
$10 \%$ PAni & 0.074 & 0.012 & 0.076 & 0.013 & $1.3-7.6 \%$ \\
$15 \%$ PAni & 0.066 & 0.019 & 0.071 & 0.022 & $4.6-13.6 \%$ \\
\hline aSV measurement by developed & sensor; b bing AAS & method for \\
confirmation.
\end{tabular}

a deviation of $3-5 \%$. In a smaller and narrower range, 10$20 \mathrm{ppb}$, the accuracy was lowered but the deviations of about $10 \%$ are acceptable.

Along with the observation for adsorption ability, the desorption behavior of 5\% PAni/MNP nanocomposite was also evaluated. Each desorption step was performed in 24 hours in a free-arsenite aqueous solution of $\mathrm{pH}=3$. After 4 times of desorption, the 5\%-PAni/MNP nanocomposite remained its high adsorption ability of $82.74 \%$ As(III) ions uptaken. Desorption ratios of $50 \%$ experienced 5 times of operation (Table 2).

With the result, this PAni/MNP nanocomposite can be recycled for at least 4 times in reality. Additionally, the released $\mathrm{Fe}$ concentration after each adsorption/desorption step was compared between 5\% PAni/MNPs and bare MNPs as corrosion indicator. In the case of nanocomposite, the released amounts of $\mathrm{Fe}$ tended to decrease, while those tended to increase in the case of bare MNPs (Table 2). At 
TABLE 2: Adsorption/desorption behavior of 5\%-PAni/MNPs (1 g) toward the initial $11 \mathrm{As}(\mathrm{III})$ solution $0.1 \mathrm{ppm}$ after adsorbing and washing 5 times, at $25^{\circ} \mathrm{C}$ and agitation.

\begin{tabular}{|c|c|c|c|c|}
\hline \multirow{2}{*}{ Try } & \multirow{2}{*}{$\begin{array}{c}\text { Adsorbed } \\
\text { As } \%(a)\end{array}$} & \multirow{2}{*}{$\begin{array}{c}\text { Released } \\
\text { As } \%^{(b)}\end{array}$} & \multicolumn{2}{|c|}{ Released $\mathrm{Fe}(\mathrm{mg} / \mathrm{L})^{(\mathrm{c})}$} \\
\hline & & & Bare MNPs & PAni/MNPs \\
\hline 1 & 93.79 & 55.25 & 45.5 & 20.5 \\
\hline 2 & 92.61 & 53.27 & 61.3 & 15.7 \\
\hline 3 & 88.22 & 49.72 & 100.7 & 11.8 \\
\hline 4 & 82.74 & 51.16 & 255.2 & 10.5 \\
\hline 5 & 77.63 & 49.20 & 382.8 & 10.7 \\
\hline
\end{tabular}

such a low $\mathrm{pH}$ value equal to 3, PAni showed good protection for MNPs as expected. The released amount of Fe from the nanocomposite might closely correspond to the physically adsorbed Fe during synthesis. In the acidic medium, MNPs were degraded; therefore, irons were found to be easily released (Table 2, (c)). In the nanocomposite forms, MNPs were covered by PAni, the solving process of iron by acidic medium was declined, and as a result, the released amounts of iron approached the constant after several recycle times (Table 2, (c)).

\section{Conclusions}

The adsorbent material was a nanocomposite prepared by coating of nanosized magnetite (MNPs) with polyaniline (PAni) in direct oxidative polymerization. The PAni/MNP nanocomposite exhibited better ability in arsenite removal than the bare magnetite. The polyaniline coating showed advantages in reducing not only agglomeration but also corrosion of MNPs and accordingly helped the adsorbent to maintain its adsorbing ability over several recycling times. The maximum adsorption ratio of the nanocomposite was obtained at $50 \mathrm{mg} / \mathrm{g}$ with aniline composition of $5 \mathrm{wt} \%$. A compact ASV-based sensing system was developed to directly evaluate the performance of adsorbent versus arsenite concentration. Using a self-fabricated triple-gold electrode, a calibration curve was built with limit-of-detection (LOD) as low as $10 \mathrm{ppb}$ As(III). The design of this study can provide a real-time/rapid treatment and observation for highly toxic As(III) contamination in water resources following the recommended standard of the World Health Organization (WHO).

\section{Data Availability}

The data used to support the findings of this study are included within the article.

\section{Conflicts of Interest}

The authors declare that there is no conflict of interest regarding the publication of this paper.

\section{Acknowledgments}

This research is funded by Vietnam National Foundation for Science and Technology Development (NAFOSTED) under grant number 103.02-2015.44. This research is supported by The World Academy of Sciences (TWAS) under Grant no. (16-169 RG/PHYS/AS_I-FR3240293345).

\section{References}

[1] A. H. Smith, C. Hopenhayn-Rich, M. N. Bates et al., "Cancer risks from arsenic in drinking water," Environmental Health Perspectives, vol. 97, pp. 259-267, 1992.

[2] J. B. Mowry, D. A. Spyker, D. E. Brooks, N. McMillan, and J. L. Schauben, "2014 annual report of the American Association of Poison Control Centers' National Poison Data System (NPDS): 32nd annual report," Clinical Toxicology, vol. 53, no. 10, pp. 962-1147, 2015.

[3] M. F. Hughes, B. D. Beck, Y. Chen, A. S. Lewis, and D. J. Thomas, "Arsenic exposure and toxicology: a historical perspective," Toxicological Sciences, vol. 123, no. 2, pp. 305-332, 2011.

[4] R. L. Kingston, S. Hall, and L. Sioris, "Clinical observations and medical outcome in 149 cases of arsenate ant killer ingestion," Journal of Toxicology Clinical Toxicology, vol. 31, no. 4, pp. 581-591, 1993.

[5] R. C. Dart, Medical Toxicology, Lippincott, Williams \& Wilkins, 3rd edition, 2004.

[6] D. Postma, F. Larsen, N. T. Thai et al., "Groundwater arsenic concentrations in Vietnam controlled by sediment age," Nature Geoscience, vol. 5, no. 9, pp. 656-661, 2012.

[7] P. L. Smedley, D. G. Kinniburgh, D. M. J. Macdonald et al., "Arsenic associations in sediments from the loess aquifer of La Pampa, Argentina," Applied Geochemistry, vol. 20, no. 5, pp. 989-1016, 2005.

[8] A. K. Sakira, I. T. Somé, E. Ziemons et al., "Determination of arsenic (III) at a nanogold modified solid carbon paste electrode," Electroanalysis, vol. 27, no. 2, pp. 309-316, 2015.

[9] P. Niedzielski and M. Siepak, "Analytical methods for determining arsenic, antimony and selenium in environmental samples," Polish Journal of Environmental Studies, vol. 12, no. 6, pp. 653-667, 2003.

[10] B. D. McKean and D. A. Gough, "A telemetry-instrumentation system for chronically implanted glucose and oxygen sensors," IEEE Transactions on Biomedical Engineering, vol. 35, no. 7, pp. 526-532, 1988.

[11] J. L. Lai, H. N. Wu, H. H. Chang, and R. J. Chen, "Design a portable bio-sensing system for glucose measurement," in 2011 International Conference on Complex, Intelligent, and Software Intensive Systems, pp. 71-76, Seoul, South Korea, June-July 2011.

[12] G. Hanrahan, D. G. Patil, and J. Wang, "Electrochemical sensors for environmental monitoring: design, development and applications," Journal of Environmental Monitoring, vol. 6, no. 8, p. 657, 2004.

[13] R. Feeney and S. P. Kounaves, "On-site analysis of arsenic in groundwater using a microfabricated gold ultramicroelectrode array," Analytical Chemistry, vol. 72, no. 10, pp. 2222-2228, 2000.

[14] X. Dai, O. Nekrassova, M. E. Hyde, and R. G. Compton, "Anodic stripping voltammetry of arsenic (III) using gold 
nanoparticle-modified electrodes," Analytical Chemistry, vol. 76, no. 19, pp. 5924-5929, 2004.

[15] K. Raj, B. Moskowitz, and R. Casciari, "Advances in ferrofluid technology," Journal of Magnetism and Magnetic Materials, vol. 149, no. 1-2, pp. 174-180, 1995.

[16] J. L. Wilson, P. Poddar, N. A. Frey et al., "Synthesis and magnetic properties of polymer nanocomposites with embedded iron nanoparticles," Journal of Applied Physics, vol. 95, no. 3, pp. 1439-1443, 2004.

[17] X. Batlle and A. Labarta, "Finite-size effects in fine particles: magnetic and transport properties," Journal of Physics D: Applied Physics, vol. 35, no. 6, pp. R15-R42, 2002.

[18] A. K. Gupta and M. Gupta, "Synthesis and surface engineering of iron oxide nanoparticles for biomedical applications," Biomaterials, vol. 26, no. 18, pp. 3995-4021, 2005.

[19] Y. S. Kang, S. Risbud, J. F. Rabolt, and P. Stroeve, "Synthesis and characterization of nanometer-size $\mathrm{Fe}_{3} \mathrm{O}_{4}$ and $\gamma-\mathrm{Fe}_{2} \mathrm{O}_{3}$ particles," Chemistry of Materials, vol. 8, no. 9, pp. 22092211, 1996.

[20] D. K. Kim, Y. Zhang, W. Voit, K. V. Rao, and M. Muhammed, "Synthesis and characterization of surfactant-coated superparamagnetic monodispersed iron oxide nanoparticles," Journal of Magnetism and Magnetic Materials, vol. 225, no. 1-2, pp. 30-36, 2001.

[21] H. Qiao, F. Chen, X. Xia, Q.-F. Wei, and F.-L. Huang, "Characterization of polyaniline/ $\mathrm{Fe}_{3} \mathrm{O}_{4}$-polyacrylonitrile composite nanofibers," Journal of Fiber Bioengineering and Informatics, vol. 2, no. 4, pp. 253-258, 2010.

[22] M. R. Saboktakin, A. M. Maharramov, and M. A. Ramazanov, "Synthesis and characterization of polyaniline/poly (p-hydroxyaniline) $/ \mathrm{Fe}_{3} \mathrm{O}_{4}$ maghetic nanocomposite," Journal of NonOxide Glasses, vol. 1, no. 3, pp. 211-215, 2009.

[23] M. R. Patil, S. D. Khairnar, and V. S. Shrivastava, "Synthesis, characterisation of polyaniline- $\mathrm{Fe}_{3} \mathrm{O}_{4}$ magnetic nanocomposite and its application for removal of an acid violet 19 dye," Applied Nanoscience, vol. 6, no. 4, pp. 495-502, 2016.

[24] K. Basavaiah, Y. Pavan Kumar, and A. V. Prasada Rao, “A facile one-pot synthesis of polyaniline/magnetite nanocomposites by micelles-assisted method," Applied Nanoscience, vol. 3, no. 5, pp. 409-415, 2013.

[25] S. A. Baig, T. Sheng, C. Sun, X. Xue, L. Tan, and X. Xu, “Arsenic removal from aqueous solutions using $\mathrm{Fe}_{3} \mathrm{O}_{4}$ - $\mathrm{HBC}$ composite: effect of calcination on adsorbents performance," PLoS One, vol. 9, no. 6, article e100704, 2014.

[26] J. Raj, A. Raina, Mohineesh, and T. D. Dogra, "Direct determination of zinc, cadmium, lead, copper metal in tap water of Delhi (India) by anodic stripping voltammetry technique," E3S Web of Conferences, vol. 1, article 09009, 2013.

[27] C.-Y. Huang, M.-J. Syu, Y.-S. Chang, C.-H. Chang, T.C. Chou, and B.-D. Liu, "A portable potentiostat for the bilirubin-specific sensor prepared from molecular imprinting," Biosensors \& Bioelectronics, vol. 22, no. 8, pp. 16941699, 2007.

[28] C.-Y. Huang, "Design of a voltammetry potentiostat for biochemical sensors," Analog Integrated Circuits and Signal Processing, vol. 67, no. 3, pp. 375-381, 2011.

[29] C. Y. Huang, Y. C. Huang, T. Y. Lin, C. H. Chang, and X. F. Li, "An SOC-based portable cyclic voltammetry potentiostat for micro-albumin biosensors," in 2007 2nd IEEE Conference on Industrial Electronics and Applications, pp. 604-609, Harbin, China, May 2007.
[30] C. Leng, J. Wei, Z. Liu, J. Shi, and C. Pan, "Synthesis of polyaniline- $\mathrm{Fe}_{3} \mathrm{O}_{4}$ nanocomposites and their conductivity and magnetic properties," Journal of Wuhan University of Technology - Materials Science Edition, vol. 25, no. 5, pp. 760-764, 2010.

[31] P. Tamilarasan and S. Ramaprabhu, "Polyaniline-magnetite nanocapsules based nanocomposite for carbon dioxide adsorption," International Journal of Greenhouse Gas Control, vol. 10, pp. 486-493, 2012.

[32] K. Song, S. Lee, C. Y. Suh, W. Kim, K. S. Ko, and D. Shin, "Synthesis and characterization of iron oxide nanoparticles prepared by electrical explosion of $\mathrm{Fe}$ wire in $\mathrm{Ar}-\mathrm{O}_{2}$ gas mixtures," Materials Transactions, vol. 53, no. 11, pp. $2056-$ 2059, 2012.

[33] Y. Dong, Y. Zhou, Y. Ding, X. Chu, and C. Wang, "Sensitive detection of $\mathrm{Pb}(\mathrm{II})$ at gold nanoparticle/polyaniline/graphene modified electrode using differential pulse anodic stripping voltammetry," Analytical Methods, vol. 6, no. 23, pp. 93679374, 2014.

[34] K. Nakamoto, Infrared and Raman Spectra of Inorganic and Coordination Compounds, John Wiley \& Sons, Inc., Hoboken, NJ, USA, 2008.

[35] C.-H. Liu, Y.-H. Chuang, T.-Y. Chen et al., "Mechanism of arsenic adsorption on magnetite nanoparticles from water: thermodynamic and spectroscopic studies," Environmental Science \& Technology, vol. 49, no. 13, pp. 7726-7734, 2015.

[36] C. Yang, J. Du, Q. Peng et al., "Polyaniline/ $\mathrm{Fe}_{3} \mathrm{O}_{4}$ nanoparticle composite: synthesis and reaction mechanism," The Journal of Physical Chemistry B, vol. 113, no. 15, pp. 5052-5058, 2009. 


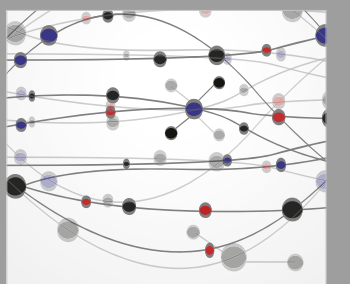

The Scientific World Journal
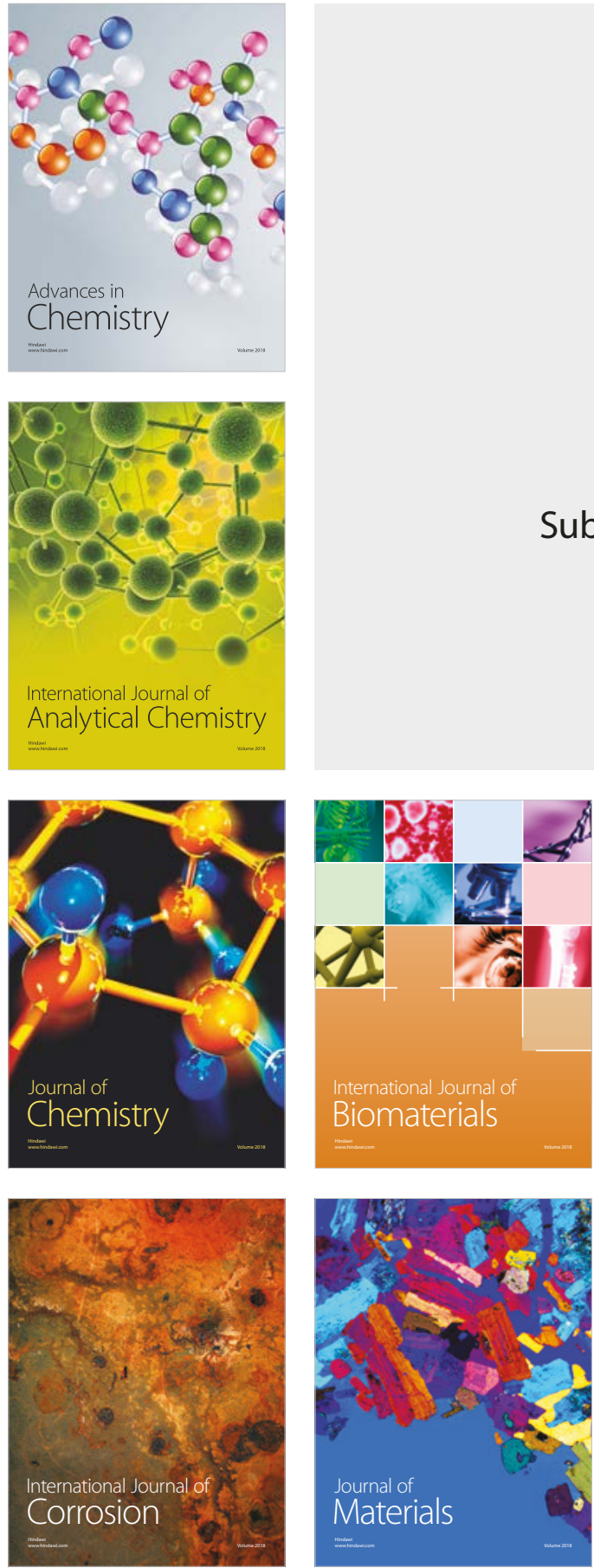

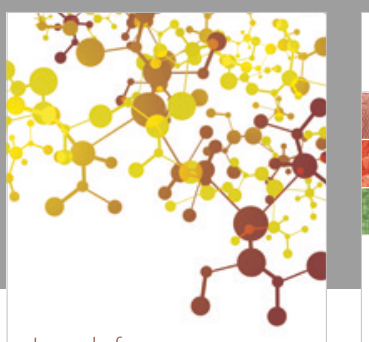

Journal of

Applied Chemistry
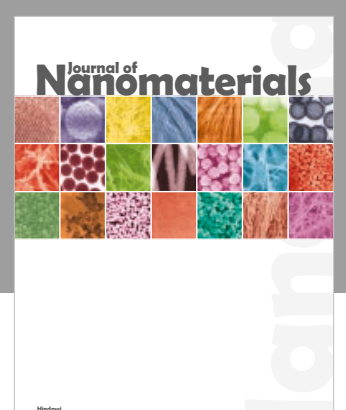

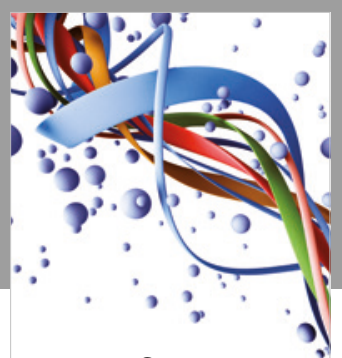

Scientifica

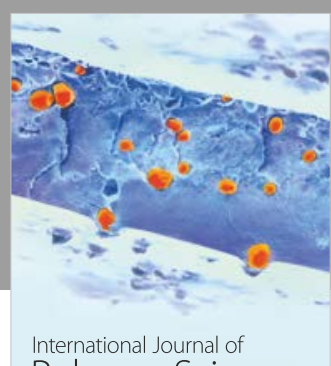

Polymer Science

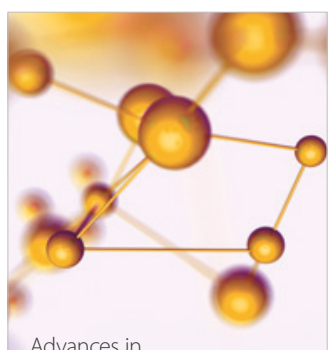

Physical Chemistry
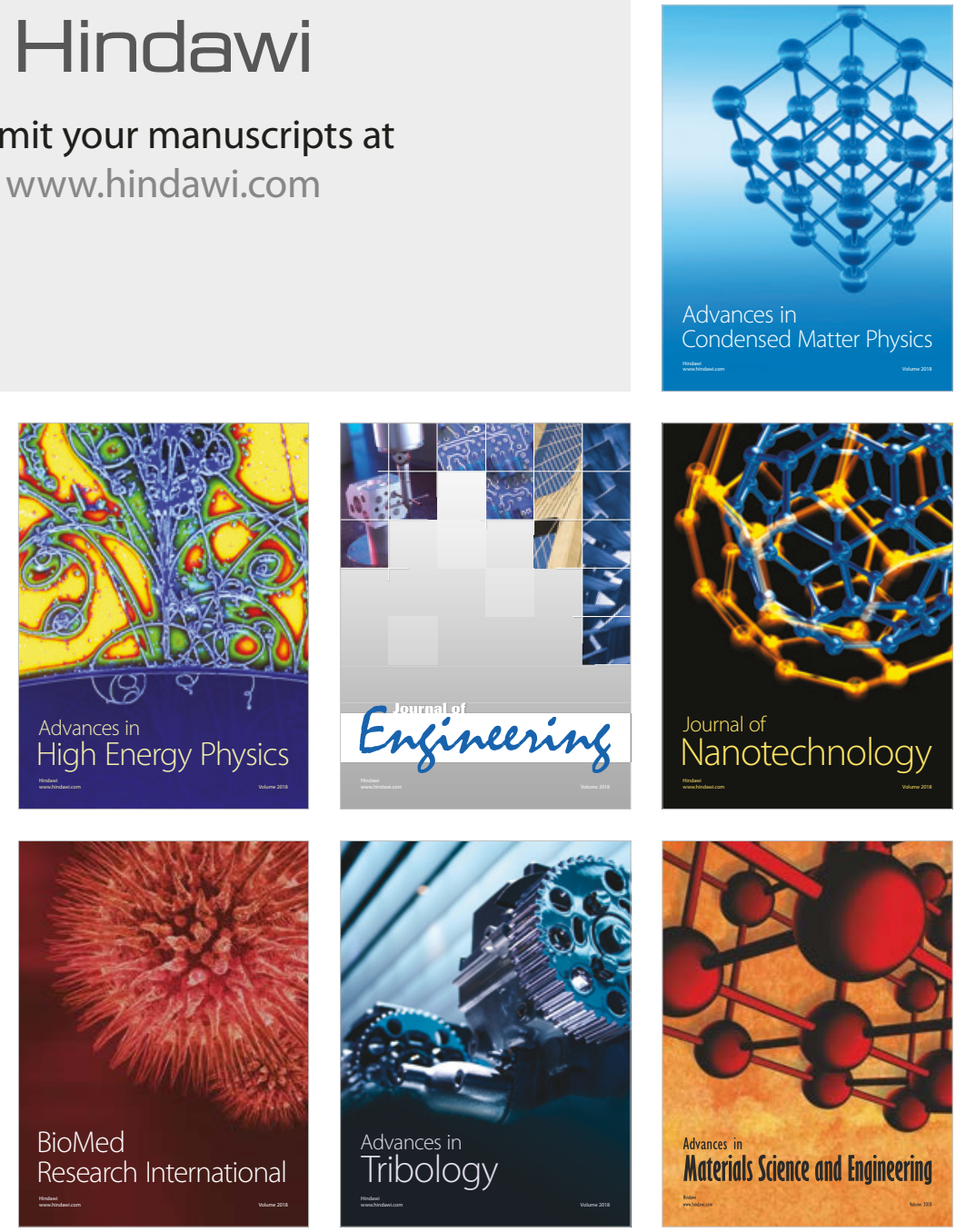